low expression of AZGP1 was also associated with progression to metastatic disease and with prostate cancer-specific mortality.

Henshall and colleagues used immunohistochemical methods to measure AZGP1 mRNA in malignant epithelium from prostatectomy specimens, obtained from 228 men with prostate cancer who underwent radical prostatectomy. Data on PSA doubling time were available for 68 of 99 men who experienced biochemical recurrence. In 17 men who had a short PSA doubling time, that was associated with local recurrence, metastasis, or death from prostate cancer, and with distant metastases $(P<0.001$ for both associations). Low or absent AZGP1 expression was present in 13 of these 17 men.

As only one-third of prostate cancer patients who experience biochemical recurrence progress to clinical disease, care should be taken when considering exposing patients to aggressive treatment. Clinical assessment of AZGP1 expression following radical prostatectomy could potentially identify patients at high risk of metastatic progression and might assist in the selection of patients for clinical trials. This study supports further testing of a simple automated histochemical assay for detecting AZGP1 levels that could be used in the clinic in the future.

Henshall SM et al. (2006) Zinc-alpha2-glycoprotein expression as a predictor of metastatic prostate cancer following radical prostatectomy. J Natl Cancer Inst 98: 1420-1424

\section{PSA-based definitions of biochemical failure influence relapse-free survival estimates}

Biochemical failure is defined differently for patients with localized prostate cancer treated with radical prostatectomy or radiotherapy, which has confounded comparison of these treatment modalities. In radiotherapy-treated patients, the standard definition of biochemical failure requires three successive PSA increases after a nadir, but a PSA increase of $2 \mathrm{ng} / \mathrm{ml}$ above nadir has been proposed as an alternative. Biochemical failure after radical prostatectomy is defined as PSA $>0.2 \mathrm{ng} / \mathrm{ml}$.

Kupelian et al. evaluated the effect of these different definitions on the estimation of biochemical relapse-free survival in 2,516 consecutive patients treated for localized prostate cancer with radical prostatectomy $(n=1,467)$ or external-beam radiotherapy $(n=1,049)$. In radiotherapy-treated patients, the alternative definition gave a $13 \%$ greater biochemical relapse-free survival estimate at 5 years, but a $12 \%$ lower estimate at 10 years, compared with the standard definition. Higher radiotherapy doses were associated with better outcomes irrespective of the definition used. After adjustment for confounding variables, radical prostatectomy was associated with better biochemical relapse-free survival than radiotherapy only with the standard definition.

Kupelian and colleagues note that the alternative definition could delay initiation of salvage therapy, because it underestimates early treatment failures compared with the standard definition. The observation that these definitions reached different conclusions on the relative efficacies of radiotherapy and radical prostatectomy could confuse clinicians and patients who are considering treatment options. Ultimately, data on mortality and distant metastases will be required to determine which definition is most accurate.

Original article Kupelian PA et al. (2006) Use of different definitions of biochemical failure after external beam radiotherapy changes conclusions about relative treatment efficacy for localized prostate cancer. Urology 68: 593-598

\section{ERSPC Rotterdam: screening detects prostate cancers with favorable characteristics}

Van der Cruijsen-Koeter and colleagues have reported the tumor characteristics and prognostic factors of prostate cancers detected in two successive screening rounds of the European Randomized Study of Screening for Prostate Cancer (ERSPC), Rotterdam section.

This multicenter, randomized, controlled trial involved 42,376 men $(21,210$ in the screening arm and 21,166 in the control arm). Men in the screening arm underwent initial evaluation from December 1993 to May 2003; the second evaluations were completed by March 2004 (an interval of 4 years). Evaluations comprised PSA measurement, digital rectal examination (DRE), transrectal ultrasonagraphy (TRUS) and sextant biopsies. Interval cancers were those detected between the first and second screening rounds, which were identified by linkage with the Rotterdam Cancer Registry database.

The first screening round detected 1,038 prostate cancers; a further 433 were detected in the second round. In total, 62 interval cancers were 